\title{
Vigilância à Saúde: deve se constituir como política pública?
}

\section{Health Surveillance: should it be a public policy?}

\section{Lislaine A. Fracolli}

Professora-Doutora do Departamento de Enfermagem em Saúde Coletiva da Escola de Enfermagem da Universidade de São Paulo. Endereço: Av. Dr. Enéas de Carvalho Aguiar, 419, CEP 05403-000, São Paulo, SP, Brasil.

E-mail: lislaineœusp.br

\section{Liliam S. Faria}

Mestre em Enfermagem em Saúde Coletiva pela EEUSP. Enfermeira do Departamento de Enfermagem em Saúde Coletiva da Escola de Enfermagem da Universidade de São Paulo.

Endereço: Av. Dr. Enéas de Carvalho Aguiar, 419, CEP 05403-000, São Paulo, SP, Brasil.

E-mail: liliamsfœusp.br

\section{Juliana Guisardi Pereira}

Enfermeira. Mestre em Enfermagem em Saúde Coletiva pela Escola de Enfermagem da Universidade de São Paulo

Endereço: Av. Dr. Enéas de Carvalho Aguiar, 419, CEP 05403-000, São Paulo, SP, Brasil.

E-mail: juguisardiळyahoo.com.br

\section{Rosemara Melchior}

Enfermeira. Mestre em Enfermagem em Saúde Coletiva pela Escola de Enfermagem da Universidade de São Paulo Endereço: Av. Dr. Enéas de Carvalho Aguiar, 419, CEP 05403-000, São Paulo, SP, Brasil.

E-mail: juguisardiळyahoo.com.br

\section{Maria Rita Bertolozzi}

Livre-Docente do Departamento de Enfermagem em Saúde Coletiva da Escola de Enfermagem da Universidade de São Paulo

Endereço: Av. Dr. Enéas de Carvalho Aguiar, 419, CEP 05403-000,

São Paulo, SP, Brasil.

E-mail: mrbertolळusp.br

Projeto financiado pelo CNPq.

\section{Resumo}

Tendo em vista que a Vigilância à Saúde pode se constituir como um potente modelo para o monitoramento de condições de saúde-doença, o objetivo deste trabalho é refletir sobre a possibilidade de instituir a Vigilância à Saúde como política pública. Para tanto, apresenta-se o conceito de política pública e discorre-se a respeito das potencialidades e limites de os modelos assistenciais se transformarem em mediadores entre as dimensões técnicas e políticas. Os dados que embasam essa discussão derivam de um projeto de pesquisa, desenvolvido entre 2005/2007, sobre a proposta de Vigilância à Saúde no município de São Paulo. Tratou-se de uma pesquisa qualitativa, onde os dados foram coletados através de entrevistas e análise documental. Utilizou-se para análise dos dados a técnica de análise de conteúdo. Nesta perspectiva tomouse o município de São Paulo como base para se discutir uma proposição de Vigilância da Saúde como processo intersetorial. Conclui-se com esse trabalho que o enfrentamento das condições adversas, provocadas pela desigualdade social, deve ser considerado como objeto da área de saúde, contudo este enfrentamento tem que se partilhado por profissionais de saúde e população, investindo principalmente na intersetorialidade como tecnologia.

Palavras-chave: Vigilância à saúde; Políticas de saúde; Políticas públicas. 


\section{Abstract}

In view of the fact that Health Surveillance could be an important framework to monitor health-disease processes, the aim of this paper is to discuss the possibility of transforming Health Surveillance into a public policy. We present the concept of public policy and approach the potentialities and limits of health models becoming mediators between the technical and political dimensions. The data that underpin this discussion come from a research project, developed during 2005/2007, about Health Surveillance in the city of São Paulo, Brazil. We utilize the example of São Paulo to discuss a proposal for surveillance as an intersection between different areas. The conclusion of this study is that, in order to solve problems created by social inequities we have to understand health like a social question and make actions in health that should involve or articulate different areas of society. That is why we understand that Health Surveillance has to be treated like a public policy.

Keywords: Health Surveillance; Health Policy; Public Policy.

\section{o Cenário de Saúde Brasileiro e a Instauração da Vigilância à Saúde como Política Pública: uma breve discussão}

Para discorrer sobre a Vigilância à Saúde é necessário, primeiramente, focalizar o cenário geral da saúde dos brasileiros. No ano 2000, menos de $20 \%$ da população brasileira vivia na área rural. Verifica-se crescente tendência ao envelhecimento da população. 0 aumento da expectativa de vida não quer dizer, necessariamente, melhor qualidade de vida. Os indicadores evidenciam redução da mortalidade infantil, mas há importantes discrepâncias entre regiões. Parcela expressiva de pessoas apresenta algum grau de desgaste no corpo bio-psíquico, que se traduz em enfermidade, com impacto sobre as atividades produtiva e familiar.

O perfil epidemiológico, ao contrário do que se propalava há anos, de que o Brasil em curto período de tempo atingiria patamares diferenciados em termos de saúde-doença, com perfil de sociedade moderna, tem mostrado a coexistência de agravos que revelam o atraso e a modernidade. Convivemos com questões que encaminham para a vulnerabilidade e o desgaste dos indivíduos, assim como aquelas relacionadas à exploração inadequada e predadora dos recursos naturais, de tratamento dos resíduos, inserção precária no trabalho, quando não, a ausência do mesmo.

A taxa de fecundidade tem mostrado declínio acentuado desde a década de 1970, gerando impacto direto na estrutura etária do Brasil. Tal fato decorre de mudanças no comportamento reprodutivo das mulheres e da crescente participação do sexo feminino no mercado de trabalho, dentre outros motivos (Brasil, 2004).

A mortalidade geral apresentou redução, de 1980 a 2001, de 11,1\%, passando de 6,3 para 5,6/100o hab. A principal causa de morte, em 200o, relacionava-se às doenças do aparelho circulatório, porém, houve aumento de casos de neoplasias, que passaram do $5^{\circ}$ ao $3^{\circ}$ lugar nesse período. Em termos de mortalidade proporcional, houve queda na proporção de mortes de menores de 1 ano e aumento na proporção de óbitos na faixa de 50 anos ou mais. Dados sobre causas externas merecem destaque, por descrever situação de risco elevado da população jovem, especialmente a masculina, destacando-se os homicídios, como fruto das 
crescentes tensões decorrentes, sobretudo, dos processos de desigualdade (Brasil, 2004).

Entretanto, ao lado dessas enfermidades, ainda persistem as doenças infecciosas, como a malária, a tuberculose, as meningites, a leishmaniose visceral e tegumentar americana, a febre amarela silvestre, as hepatites virais, a esquistossomose e a leptospirose. Ao seu lado, Aids, cólera, dengue e hantaviroses são apontadas como emergentes e reemergentes (Brasil, 2004).

O Brasil experimenta melhora no acesso aos serviços básicos de saneamento, mas a meta de universalização da cobertura ainda não foi alcançada, especialmente para as instalações sanitárias, e a desigualdade na distribuição em populações rurais e urbanas ainda persiste. Há importantes diferenças territoriais, sendo as regiões Sul, Sudeste e Centro-Oeste as que apresentam maior cobertura (Brasil, 2004). A média de anos de estudo das pessoas de 25 ou mais anos apresenta uma disparidade de 4,3 anos de estudo entre a Unidade Federativa que possui a maior e a menor média de anos de estudo: respectivamente, Distrito Federal e Piauí. Nenhum estado brasileiro enquadrase nos ideais de instrução internacionalmente aceitáveis, estando mais próximos destes o Distrito Federal e alguns estados das regiões Sul e Sudeste: há um abismo educacional entre as várias regiões do País (Brasil, 2004).

Isso também ocorre em relação à distribuição de estabelecimentos de saúde, que é marcadamente diferenciada entre as regiões e os estados brasileiros. Há, também, desigualdade na oferta de empregos de médicos e enfermeiros, com maior concentração nas regiões Sul e Sudeste, as quais agregam $57 \%$ da população brasileira (Brasil, 2004).

Essa realidade coloca novas necessidades no âmbito dos serviços de saúde, que requerem ações de acompanhamento terapêutico aos agravos já instalados, assim como o monitoramento de potenciais de desgaste. É nesse sentido que o estabelecimento de políticas continuadas que levem em conta as necessidades de manutenção do fortalecimento dos sujeitos, assim como o monitoramento e o alerta continuado em relação a situações que podem induzir à vulnerabilidade, são importantes focos para a instauração de políticas de saúde.

Mas, o que são políticas? E o que são políticas pú- blicas? Política refere-se à consciência da historicidade, ao processo de construção de projetos no plano em que emergem contradições sociais. A política reivindica capacidade reflexiva sobre a história que se coloca como dialética que se configura, por um lado, por um sujeito social, por seus projetos e suas práticas e, por outro, pela realidade como campo de estruturas sociais, institucionais e de relações entre forças em luta no sentido de fazer de suas utopias, realidade (Belmartino, 1994).

Segundo Costa (1998), a política pública refere-se ao espaço de tomada de decisão autorizada ou sancionada por intermédio de atores governamentais, compreendendo atos que viabilizam agendas de inovação em políticas ou que respondem a demandas de grupos de interesse. A configuração de uma política é função direta das capacidades da unidade, associação e barganha entre indivíduos, grupos e associações de interesses, tendo como alvo as decisões alocativas das agências governamentais.

Sob a égide do capitalismo, o Estado planeja e executa, por meio de uma estratégia econômica central, o interesse capitalista coletivo. 0 Estado é a expressão simultânea de inúmeras tendências contraditórias, é um lugar organizacional específico investido de autoridade de intervir e de poder decisório. 0 reconhecimento do Estado como expressão dos interesses de classe é insuficiente se não for considerado um segundo papel como organização: como uma unidade corporativa e, ainda, pode se apresentar como guardião dos interesses universais (Costa, 1998).

No início da década de 1990, com o processo de Reforma Sanitária, vivencia-se uma tentativa de reinstitucionalização das políticas públicas de proteção. Esta, entretanto, desenvolveu-se de forma bastante desigual nas diversas áreas de políticas. Segundo Costa (1998), a agenda da dívida social pareceu ser muito mais retórica ou depositária de uma crença absoluta nas soluções de políticas emergenciais de combate à pobreza do que, de fato, uma proposta de nova configuração para o sistema de proteção social do país.

Com o Sistema Único de Saúde (SUS), verifica-se um novo padrão institucional de proteção social. Além da universalização do acesso, o SUS propôs a integralidade da atenção, antes assegurada apenas ao segmento da população incorporada ao mercado formal de trabalho. 


\section{o Modelo de Vigilância à Saúde como Mediação entre o Técnico e o Político}

Desde o início do processo de construção e implantação do SUS é debatida a proposta da Vigilância à Saúde, devido à necessidade de se reorganizar e reorientar os serviços de saúde e de ampliação das ações de vigilância epidemiológica, no sentido de contemplar os problemas de saúde e condições de vida em sua amplitude, uma vez que essas ações sempre estiveram voltadas para o controle do hospedeiro, agente etiológico e meio ambiente (IESUS, 1993; Bertolozzi e Fracolli 2004).

Em revisão da literatura verifica-se que Vigilância à Saúde é amplamente discutida, sendo levantadas diversas proposições com diferentes terminologias: vigilância ‘à' saúde; vigilância 'em’ saúde; vigilância em saúde pública e vigilância 'da' saúde, no entanto, com o eixo em comum na epidemiologia e informação em saúde. Ao longo da década de 1990 até a atualidade, apresentou-se uma variada produção científica acerca da Vigilância à Saúde em que, progressivamente, amplia-se e redefine-se o objeto da Vigilância Epidemiológica, com evolução do conceito de vigilância chegando-se à conformação de um modelo de atenção à saúde. Sumariamente destacam-se, a seguir, três principais noções de Vigilância à Saúde que balizam os demais estudos, os quais contribuem com a especificação de instrumentos e meios para sua operacionalização, e com reflexões sobre as potencialidades de tal modelo (Faria, 2007).

- A Vigilância à Saúde se propõe a integrar a vigilância epidemiológica e a vigilância sanitária a fim de superar a fragmentação das ações de vigilância no âmbito municipal. Têm fortalecido, em particular, as ações de vigilância sanitária, viabilizando a articulação com centros de saúde do trabalhador, constituindo-se em espaço privilegiado para a implementação de campanhas de vacinação e controle de epidemias. Mais recentemente, essa proposição parece transcender o âmbito da vigilância epidemiológica e sanitária, organizando-se a partir da integração coordenada das áreas de vigilância ambiental, de saúde do trabalhador e de zoonoses, com o objetivo final de contribuir para a prevenção de agravos e a promoção da saúde, a exemplo da "Coordenadoria de Vigilância em Saúde", implantada através da Secretaria de Saúde do Município de São Paulo (Barata, 1993; Tambellini e Câmara 2002; Neves e Alves, 2005).

- A ampliação do objeto da vigilância epidemiológica, em que se parte da análise de situações de saúde de grupos populacionais e considerando-se suas condições de vida, foi formulada como um instrumento para possibilitar a investigação, monitorização e montagem de bancos de dados sobre outros agravos à saúde, além das doenças transmissíveis (Waldman, 1993).

- A Vigilância à Saúde é proposta como instrumento para a transformação dos determinantes de saúde e doença referenciada pelo conceito de território, participação da população e promoção da saúde. Conforme Mendes (1995), para apreender um recorte dos aspectos de um dado território, é necessário que se reúnam informações demográficas, socioeconômicas, políticoculturais, epidemiológicas e sanitárias. A partir daí, torna-se possível identificar e analisar os problemas, os perfis epidemiológicos e as necessidades de saúde das pessoas, para definir prioridades de atenção à saúde, com base na intersetorialidade, na integralidade e na eqüidade. Dessa forma, a Vigilância à Saúde opera com base em sistemas geográficos de informação, a partir da microlocalização de problemas, e visa o planejamento local em saúde (Mendes, 1995). Incorporando o conceito de processo de trabalho em saúde à Vigilância à Saúde, esta se constitui num modelo assistencial destinado a superar a dicotomia entre as práticas coletivas e individuais, que tem como objeto os modos de vida dos diferentes grupos sociais e as diversas expressões do processo saúde-doença. 0 referido modelo toma como principais características: intervenção sobre problemas de saúde em seus diferentes períodos do processo saúde-doença; ênfase em problemas que requerem atenção e acompanhamento contínuos; operacionalização do conceito de risco; articulação entre as ações promocionais, preventivas e curativas; atuação intersetorial e ações sobre o território (Teixeira e col., 1998).

A partir desse referencial, sobressaem-se como potencialidades da Vigilância à Saúde: o monitoramento de condições de vida e saúde; a reorientação dos serviços de saúde na superação das desigualdades de cobertura, acesso e qualidade do controle social da informação em saúde. 
Bastos (2005) aponta que a construção de um sistema de vigilância de doenças e agravos não-transmissíveis, além de produzir e divulgar informações epidemiológicas sobre tais eventos e as formas de prevenção, relacionadas à rede de promoção da saúde, deve articular-se com a assistência, compondo sistemas de avaliação de serviços. As fontes de informação podem ser variadas, incluindo desde prontuários de usuários dos serviços de saúde e registros de exames de laboratório, até questionários de inquéritos de situações de saúde e observações diretas de fatos e eventos. Já uma única fonte integradora de todos os dados relevantes dos usuários do sistema de saúde ou da comunidade da área de abrangência do serviço de saúde facilita o uso de sistema de informação (Mota e Carvalho, 1999). O SIAB constitui-se em uma importante fonte integradora de dados gerados na Atenção Básica à Saúde.

Martins e Fontes (2004) incorporam o conceito de redes sociais à área de Vigilância à Saúde, a qual compreenderia o processo saúde-doença na coletividade e transcenderia as ações burocráticas para alcançar um processo que envolve a participação da população, do governo, de organizações civis e científicas. Os autores defendem que a construção de Redes de Vigilância pode ser útil no apoio ao desenvolvimento de ações solidárias de conhecimento, detecção e prevenção de epidemias, numa perspectiva que acolha integralmente a idéia de ambiente aos sistemas social e coletivo. Com a participação da comunidade, as Redes de Vigilância podem proporcionar o controle social e permitir a identificação de nós críticos que obstaculizam os fluxos comunicativos entre as instituições públicas e a sociedade civil. Nessa perspectiva, destaca-se o potencial para a mobilização de diversos atores em diferentes instâncias, o que permitiria maior eficácia e transparência aos programas epidemiológicos do SUS. Para tanto, destacam que é essencial disponibilizar amplamente as informações e utilizar a educação em saúde nos microambientes sociais (Martins e Fontes, 2004). Além disso, pode-se desenvolver o controle social da informação sobre os determinantes de saúde pela população através da supervisão das políticas e ações nas esferas econômica, social, política e cultural e a mobilização social, no sentido da promoção da saúde (Freitas, 2003).
A Vigilância à Saúde, no Brasil, tem sido traduzida para a forma de política pública e isso pode ser evidenciado estruturalmente pela criação, no âmbito do Ministério da Saúde, de uma Secretaria de Vigilância à Saúde (Brasil, 2004). Essa secretaria, além de desenvolver as ações tradicionais da Vigilância Epidemiológica e controle de agravos transmissíveis, amplia suas ações para os agravos não-transmissíveis, para a promoção da saúde e para projetos específicos sobre indicadores de saúde. Seguindo essa estrutura organizacional, muitos Estados e municípios no Brasil têm criado estruturas técnico-políticas de Vigilância em Saúde.

\section{A Vigilância à Saúde no Município de São Paulo: à guisa de exemplo}

As discussões apresentadas a seguir derivam de projeto de pesquisa desenvolvido entre $2005 / 2007^{1}$, o qual teve como objetivos descrever e analisar a Vigilância à Saúde, num Distrito de Saúde Escola (DSE) do Município de São Paulo, e identificar os momentos de intersecção entre a academia e o serviço que contribuem para a implementação da Vigilância à Saúde. Tal distrito foi escolhido, pois partiu-se da hipótese de que a convivência entre atores da Universidade e do serviço de saúde poderia resultar em parcerias que contribuíssem para redefinição do modelo assistencial no sentido da Vigilância à Saúde. Os dados descritos a seguir derivam de entrevistas com profissionais e gerentes de Unidades Básicas de Saúde (UBS) da região centro-oeste do município, em que uma parte foi submetida à análise segundo o Discurso do Sujeito Coletivo (Lefèvre e col., 200o), e com a outra parte procedeu-se à análise de Discurso orientada pela Teoria Gerativa de Discurso. Além disso, analisaram-se documentos publicados pela Secretaria de Saúde do Município de São Paulo (SSMSP).

Em 200o, o Município de São Paulo fez a opção por instituir um sistema municipal de Vigilância à Saúde, apoiado na necessidade de estruturar um sistema com grande competência de nível central para coordenar todo o sistema de vigilância e para executar complementarmente as atividades de difícil realização pelos organismos de nível local (Neves, 2003). Criouse, então, a COVISA (Coordenadoria de Vigilância à

1 Projeto financiado pelo CNPq. 
Saúde) que se pretendia que fosse uma autarquia municipal capaz de trabalhar com autonomia nas questões de vigilância. Coerentemente, com todo movimento de descentralização do SUS e da proposta das subprefeituras, propôs-se também estruturar serviços que dariam conseqüência às ações de vigilância no plano local, através da instituição das SUVIS (Superintendência de Vigilância à Saúde) (Neves, 2003).

A COVISA abarca em seu interior o Centro de Prevenção e Controle de Doenças (CCD), a Vigilância Epidemiológica, a Vigilância em Saúde Ambiental, a Vigilância de Produtos, Serviços e Saúde do Trabalhador e a Vigilância Sanitária. A COVISA se responsabiliza por coordenar as ações de vigilância, prevenção e controle de determinados agravos, através das subgerências de Imunização, de Doenças Transmissíveis e de Doenças e Agravos Não-Transmissíveis. No nível regional, esse trabalho se dá através da parceria com as SUVIS, articuladas às Subprefeituras, cujas equipes foram capacitadas logo no início da gestão e continuam sendo alvo de capacitação continuada(Neves, 2003).

Basicamente, as principais atribuições da SUVIS são: participação do Sistema Nacional de Vigilância Epidemiológica das Doenças de Notificação Compulsória, participação no Programa Nacional de Imunização (PNI) e estudo da ocorrência, distribuição e determinantes de agravos não-transmissíveis para a proposição de medidas de prevenção primária (Prefeitura Municipal de São Paulo, 2008).

Segundo Neves (2003), o sistema de Vigilância no Município de São Paulo está sendo pensado considerando-se os princípios norteadores dos Distritos, destacando-se que suas competências vão além de cuidar da atenção aos indivíduos doentes, pensando-se, também, nos condicionantes de adoecimento e qualidade de vida, além de articular e gerir os recursos públicos disponíveis para a melhoria da qualidade de vida nos Distritos. Vinculado à SMSSP, encontramos o Ceinfo (Centro de Informações para a Saúde), que funciona como uma central de inteligência epidemiológica, possuindo todos os tipos de dados sobre morbidade, mortalidade, produção e produtividade de serviços de saúde, entre outros. O Ceinfo e a Covisa entendem que a diferença entre o trabalho que cada um desenvolve deve-se ao fato de que o Ceinfo gera os dados relativos à saúde e às condições de vida, ocupando-se de produzir informações e indicadores, mas não de avaliá-los e intervir sobre eles, enquanto a Covisa se utiliza dos mesmos para monitorar e intervir nas condições de saúde, tendo um papel mais de detecção e intervenção em problemas de saúde.

A contribuição mais recente da Ceinfo para a Covisa e a SMSSP é o "painel de monitoramento", construído com recursos do Vigisus. O Painel de Monitoramento das Condições de Vida e Saúde e da Situação dos Serviços de Saúde é um amplo e sintético panorama da atuação dos serviços de saúde municipais e dos seus principais problemas. Ele foi criado para subsidiar os gestores municipais com um instrumento de acompanhamento da sua prática cotidiana e avaliação das ações sob sua coordenação. Constitui-se, ainda, num instrumento de democratização da informação em saúde ao permitir o compartilhamento das avaliações realizadas com os conselhos de saúde dos diversos níveis da SMSSP. A proposta busca contemplar as prioridades do SUS definidas na política nacional e estadual, e especialmente na municipal, produzindo um elenco de indicadores que contribuam para informar sobre o andamento dos projetos e ações de saúde no município.

O processo de construção do Painel partiu da sistematização e avaliação de indicadores existentes ou exigidos no Pacto da Atenção Básica (PAB), na Programação Pactuada Integrada (PPI) e nos Indicadores de Avaliação da Gestão (IAG) do Estado de São Paulo. Num segundo momento, buscou-se suprir lacunas incorporando os demais projetos da SMS e estabelecendo indicadores para metas dos projetos prioritários da gestão municipal.

Através do painel, pode-se identificar a existência de um suporte relativo a dados sobre saúde e vida, que facilita o desenvolvimento de propostas de Vigilância à Saúde no município, pois, uma vez tendo centrais capazes de gerar dados sobre vida e saúde, o trabalho dos profissionais da Vigilância à Saúde pode se concentrar na avaliação mais qualitativa das informações e na organização de propostas mais efetivas de intervenção, por Distrito ou macro-região.

Com esta descrição, é possível observar em que base está organizada a estrutura teórico-operacional da Vigilância à Saúde no município de São Paulo. Sabese, contudo, que o trabalho em saúde tem certa flexibilidade organizacional em função dos atores e dos problemas de saúde específicos de cada região. 


\section{A Vigilância à Saúde: a prática nas unidades básicas de saúde de uma região do município de São Paulo}

Na construção da Vigilância à Saúde, conforme pôde ser evidenciado através de entrevistas com os trabalhadores da Atenção Básica e da Supervisão de Vigilância em Saúde, prevalecem ainda práticas de vigilância fortemente relacionadas à vigilância epidemiológica (VE). Pode-se verificar que o objeto do trabalho da vigilância focaliza-se na doença/agravo de saúde, substantivamente, nas doenças transmissíveis.

Por outro lado, outras práticas citadas como realizadas e que parecem transcender a proposta da Vigilância Epidemiológica clássica, numa perspectiva de Vigilância à Saúde, são:

- Construir indicadores e metas que subsidiarão o monitoramento das atividades e seus respectivos resultados. Com a ressalva de que é necessário se aproveitar dos dados já produzidos e que, portanto, não requeiram "invenção", a exemplo de alguns indicadores que tiveram como meta a melhoria da cobertura vacinal, a cobertura do pré-natal, a diminuição da gravidez na adolescência, dentre outros.

- "Pensar" o território mais amplamente, configurandoo como um espaço onde as pessoas vivem e apresentam necessidades, e assim conduzir ações de vigilância no formato de projetos a partir da detecção de problemas prioritários na área de abrangência da UBS (Faria, 2007).

Considera-se muito interessante que esses tipos de práticas tenham sido apontadas, ainda que como horizontes a serem alcançados, o que revela um adensamento nas práticas de vigilância, que antes se limitavam ao preenchimento de "boletim de informação" sobre as notificações semanais. Além disso, observa-se que a implementação do Programa Saúde da Família estimula práticas ampliadas de vigilância. A esse respeito, considera-se que a coleta de dados, o preenchimento do Sistema de Informação da Atenção Básica (SIAB), a discussão sobre as informações coletadas pelos agentes comunitários de saúde e a presença diária nos domicílios são atividades que contribuem para essa concepção ampliada de vigilância. Ademais, há tentativas de identificação de situações de violência no cotidiano familiar. Ora, essas ações ainda não se configuram como monitoramento das con- dições de saúde-doença no sentido de continuidade, de alerta continuado, mas permitem identificar que os trabalhadores de saúde desejam ousar para praticar ações diferentes das correntemente realizadas.

Nessa perspectiva, foi promissor encontrar algumas práticas que se orientam, ainda que de forma incipiente, à Vigilância à Saúde. Nestas, observou-se tentativas de trabalho em equipe multiprofissional debatendo, como objeto da atenção, não mais os agravos infecto-contagiosos, mas o monitoramento de agravos crônico-degenerativos e, mais ainda, de necessidades de saúde, incorporando condições de vida, mas ainda não de trabalho. Dos depoimentos dos trabalhadores da saúde emergiram significados em que se detectam indícios que se relacionariam aos determinantes de saúde não reduzidos a fatores de risco, o que possibilita o desencadeamento de ações de intervenção intersetorial. Ainda nesse âmbito, evidenciaram-se tentativas de inclusão de tecnologias de comunicação social, no formato de atividades grupais, principalmente quando se trata de doenças que requerem envolvimento da comunidade, por exemplo, mutirões de limpeza de território, em caso de dengue.

Sobre as dificuldades sentidas pelos trabalhadores para implementar a Vigilância à Saúde destaca-se a insuficiente qualificação profissional, o que se agrava com a complexidade desse campo, em que os profissionais dificilmente têm domínio de todas as suas esferas. De modo geral, os pontos de estrangulamento citados foram: a falta de capacitação em serviço para integrar a vigilância e a assistência, viabilizando o monitoramento e a avaliação das ações; além da falta de domínio de conhecimento sobre o território de atuação da UBS.

Num outro pólo, sobressai-se a falta de conhecimento entre os usuários dos serviços de saúde a respeito do SUS, bem como da função e relevância das ações de vigilância, o que culmina na não participação da comunidade na reivindicação da realização de ações nesse âmbito.

\section{A Interface Ensino-Serviço na Construção da Vigilância à Saúde}

A participação da Universidade na construção do modelo assistencial da Vigilância à Saúde, sob a perspectiva dos trabalhadores, tem se dado pela implanta- 
ção de ações na UBS, como resultado das ações de ensino de graduação e pela capacitação dos profissionais da rede na abordagem de temas relacionados ao Sistema Nacional de Vigilância Epidemiológica de Doenças de Notificação Compulsória e das DANTs.

Por outro lado, ressalta-se a percepção de que a participação da Universidade tem sido pequena, por falta de projeto político municipal na estruturação do modelo da Vigilância à Saúde, predominando ações de vigilância epidemiológica, notadamente pelo enfrentamento "de uma demanda que chega, mas não a uma demanda que esta por trás dos índices de morbidade, de mortalidade e dos coeficientes" (Pereira, 2007).

É importante destacar que, na perspectiva dos trabalhadores, a construção do modelo assistencial da Vigilância à Saúde deve se dar através da intersetorialidade (Pereira 2007). Considerando-se que as mudanças na formação e na atenção se dão ao mesmo tempo e alimentam-se mutuamente, Feuerwerker e colaboradores (200o) afirmam que para que o processo de mudança seja efetivo - uma vez sendo caracterizado por valores, atitudes e práticas - ele deve ser construído no espaço local, protagonizado pelos profissionais, docentes, estudantes e comunidade. Dessa forma, aponta-se para a necessidade, no local de estudo, do estabelecimento de parceiras, minimamente, entre universidade, serviços e comunidade, sendo necessário haver um processo de sensibilização e negociação que leve ao fortalecimento das articulações e à valorização dos sujeitos sociais envolvidos.

\section{Considerações Finais}

As atividades de VE tradicionalmente realizadas guardam coerência à proposta da Programação, modelo que reduz as necessidades de saúde a danos, deixando de lado as condições de reprodução social. Isso é o que Rivera (1995) chama de "permanência no plano epifenomênico da realidade”, que permite a percepção restrita aos fenômenos sanitários, não transcendendo ao seu conhecimento e explicação.

As condições objetivas de existência constituem uma forma particular de inserção no sistema produtivo de consumir, de relacionar-se com o território e de participar no processo político de tomada de decisões. A Vigilância à Saúde, dessa forma, diz respeito a um processo contínuo, que pretende dar visibilidade a essas condições objetivas e recolocá-las para ilumi- nar o território, no sentido de ajudar a emergir as necessidades de saúde-doença.

A reorganização da atenção com base na Vigilância à Saúde implica em novos papéis, novas relações e novas práticas em todos os níveis do sistema. Há que se continuar investindo em políticas de financiamento, de gestão e de remuneração, espaço que vem sendo constituído pelos gestores do SUS nos diferentes níveis, e em novas estratégias de discussão e conversa sobre o papel de todos os atores na formação profissional e na reorganização da atenção.

\section{Referências}

BARATA, R. B. Reorientação das práticas de vigilância epidemiológica. In: CARVALHO, D. M. de; MOTA, E. L. A.; TEIXEIRA, M. da G. L. C. SEMINÁRIO NACIONAL DE VIGILÂNCIA EPIDEMIOLÓGICA, 1993, Brasília. Anais... Brasília, DF: Centro Nacional de Epidemiologia, 1993.p.63-68.

BASTOS, L. G. C. Vigilância de doenças e agravos nãotransmissíveis na América Latina: o estado da arte. 0 Mundo da Saúde, São Paulo, v. 29, n. 1, p. 82-87, 2005.

BERTOLOZZI, M. R.; FRACOLLI, L. A. Vigilância à saúde: alerta continuado em saúde coletiva. O Mundo da Saúde, São Paulo, v. 28, n. 1, p. 14-20, 2004.

BELMARTINO, S. La investigación social em salud em um contexto teorico carente de certezas. In: KORNBLIT, A. L. Ciencias Sociales y Medicina: la salud em Latinoamerica. Buenos Aires: Instituto de Investigaciones de la Facultad de Ciencias Sociales de la Universidade de Buenos Aires, 1994. p. 92-104.

BRASIL. Ministério da Saúde. Secretaria de Vigilância em Saúde. Departamento de Análise de Situação de Saúde. Saúde Brasil 2004. Brasília, DF, 2004. Disponível em: http://portal.saude.gov.br/ portal/arquivos/pdf/saude_brasil_2004.pdf. Acesso em: 15 maio 2007.

COSTA, N. R. Políticas públicas, justiça distributiva e inovação: saúde e saneamento na agenda social. São Paulo: Hucitec, 1998.

FARIA, L. S. As práticas de vigilância na Supervisão Técnica de Saúde do Butantã - São Paulo (SP): perspectivas para o alcance da vigilância à saúde. 2007. Dissertação - Escola de Enfermagem da Universidade de São Paulo, São Paulo, 2007. 
FREITAS, C. M. de. A vigilância à saúde para a promoção da saúde. In: CZERESNIA, D.; FREITAS, C. M. (Org.). Promoção da saúde: conceitos, reflexões, tendências. Rio de janeiro: Fiocruz, 2003. p. 141-172.

FEUERWERKER, L.; COSTA, H.; RANGEL, M. L. Diversificação de cenários de ensino e trabalho sobre necessidade/problemas da comunidade. Divulgação em Saúde para Debate, Londrina, n. 22, p. 36-48, 2000.

IESUS - INFORME EPIDEMIOLÓGICO DO SUS. As ações de vigilância epidemiológica e controle de agravos na perspectiva do SUS. Informe Epidemiológico do SUS, Brasília, v. 2 (número especial), p. 77-95, 1993.

LEFÈVRE, F.; LEFÈVRE, A. M. C.; TEIXEIRA, J. J. V. (Org.). O discurso do sujeito coletivo: uma nova abordagem metodológica na pesquisa qualitativa. Caxias do Sul: Educs, 2000.

MARTINS, P. H.; FONTES, B. Construindo o conceito de redes de vigilância em saúde. In: MARTINS, P. H.; FONTES, B. (Org.). Redes sociais e saúde: novas possibilidades teóricas. Recife: Ed. Universitária da UFPE, 2004. p. 103-120.

MENDES, E. V. Distrito sanitário: processo social de mudanças das práticas sanitárias do SUS. São Paulo: Hucitec; Rio de Janeiro: Abrasco, 1995.

MOTA, E. L. A.; CARVALHO, D. M. Sistemas de informação em saúde. In: ROUQUAYROL, M. Z.; ALMEIDA, N. F. de. Epidemiologia e saúde. Rio de Janeiro: MEDSI, 1999. p. 505-521.

NEVES, H. Vigilância em saúde na capital paulista. In: SOUSA, M. F.; MENDES, A. Tempos radicais da saúde em São Paulo: a construção do SUS na maior cidade brasileira. São Paulo: Hucitec, 2003. p. 259300 .
NEVES, H.; ALVES, J. C. de M. Vigilância em saúde: a experiência de São Paulo. O Mundo da Saúde, São Paulo, v. 29, n. 1, p. 104-111, 2005.

PEREIRA, J. G. Articulação ensino-serviço para a construção da vigilância da saúde: em foco o Distrito do Butantã. 2007. Dissertação - Escola de Enfermagem da Universidade de São Paulo, São Paulo, 2007.

RIVERA, F. J. U. Agir comunicativo e planejamento social (uma crítica ao enfoque estratégico). Rio de Janeiro: Fiocruz, 1995.

PREFEITURA MUNICIPAL DE SÃO PAULO. Secretaria da Saúde. Supervisões de Vigilância em Saúde (SUVIS). São Paulo, SP. Disponível em: <http:// portal.prefeitura.sp.gov.br/secretarias/saude/ vigilancia_saude/organizacao/ooo4>. Acesso em: 18 mar. 2008.

TAMBELLINI, A. T.; CÂMARA, V. de M. Vigilância ambiental em saúde: conceitos, caminhos e interfaces com outros tipos de vigilância. Cadernos de Saúde Coletiva, Rio de Janeiro, v. 1o, n. 1, p. 77-93, 2002.

TEIXEIRA, C. F.; PAIM, J. S.; VILASBÔAS, A. L. SUS, modelos assistenciais e vigilância à saúde. Informe Epidemiológico do SUS, Brasília, v. 7, n. 2, p. 7-28, 1998.

WALDMAN, E. A. As concepções de vigilância como instrumento de saúde pública e a implantação do SUS. In: CARVALHO, D. M. de; MOTA, E. L. A.; TEIXEIRA, M. da G. L. C. SEMINÁRIO NACIONAL DE VIGILÂNCIA EPIDEMIOLÓGICA, 1993, Brasília. Anais. Brasília, DF: Centro Nacional de Epidemiologia, 1993. p. 45-51. 